\title{
Cephalosporin resistant bacterial strains isolated from respiratory infections
}

${ }^{1}$.Faculty of medicine, University "Ovidius" Constanta

${ }^{2}$ Emergency Clinical County Hospital "Sf. Apostol Andrei" Constanta

\begin{abstract}
Objectives: The objective of the study is the evaluation of the actual resistance to second, third, and fourth generation cephalosporins over bacterial strains isolated from respiratory infections. The main causes for cephalosporin resistance of pathogenic and conditioned pathogen bacteria are: widespread usage, and impair immune response.

Materials and methods: The analyzed specimens were throat swabs and sputum, from adult patients. The tests were performed using disk diffusion technique. We tested the following cephalosporin: From second generation: cefuroxime axetil; from third generation: cefotaxime, ceftazidime, cefpodoxime; Combinations of cephalosporins and beta-lactamase inhibitors: cefotaxime + clavulanic acid; ceftazidim + clavulanic acid; From fourth generation: cefepime; and association cefepime and clavulanic acid.

Results: The following bacterial strains were isolated: Staphylococcus aureus, Streptococcus pneumoniae, Group C $\beta$-hemolytic Streptococcus, E. coli, Klebsiella pneumoniae and Proteus sp. The Group A. $\beta$-hemolytic Streptococcus isolated strains were not tested. For Staphylococcus aureus, E. coli, K. pneumoniae and Proteus, we found a high frequency resistance to
\end{abstract}

Mihaela Botnarciuc

Str. Bogdan Voda 64, Constanta, Romania

Email:mbotnarciuc@yahoo.com cefuroxim, approximately 47\%. Highest resistance to third generation cephalosporin was identified to E.coli and Klebsiella pneumoniae, especially resistant to cefotaxime, cefotaxime + clavulanic acid and ceftazidime.

Conclusions: Cefpodoxime can be considered as a first election antibiotic in treating upper and lower respiratory tract infections, due to the lowest level of bacterial strain resistance, approximately $10 \%$ of the third generation cephalosporines tested.

Also, cefepime may be proper in treating severe respiratory tract infections, with resistant broad-spectrum antibiotics bacterial strains. In our trial, resistance to cefepime was to a minimum low, approximately $4 \%$, represented by the E.coli strains.

Keywords: cephalosporins, resistance, respiratory, infections

\section{Objectives}

The primary causes for the resistance of pathogenic and conditionally pathogenic bacteria to cephalosporins are: large-scale use of the former, as well as patients suffering from a complex pathology (especially one affecting the cardio-vascular and respiratory systems) associated with immune insufficiency [1,2]. Antimicrobial therapy against infections of the respiratory tract makes frequent use of cephalosporins, especially orally-administered 
in ambulatory care, but also parenterally, as an alternative to the amoxicillin - clavulanic acid or amoxicillin - sulbactam combinations [3,4]. It is often the case that the infectious agent is not isolated, hence no antibiogram is performed. In view of the aforementioned, based on in vitro sensitivity tests, the present study sets out to assess the current level of efficiency as shown by certain second-, third, or fourthth-generation cephalosporins against isolated bacterial strains occurring during respiratory infections.

\section{Materials and Method}

The biological products under investigation consisted of pharyngeal, nasal and sputum exudates collected from children and adult patients, who presented clinical symptoms suggestive of upper and lower respiratory tract infections.

Staphylococcus aureus is a conditionally pathogenic species. The estimation on its involvement in an infectious process based on the morphology of the growth can be conducted according to quantitative or qualitative criteria: rich, pure or prominent growth compared to the other isolated species, as well as by correlation to the morphology of the smear sampled from the pathological product (exudate, sputum), where the presence of polymorphonuclear leucocytes indicates an inflammatory process. The presence of the Staphylococcus aureus in the nasal exudate normally implies carriage; however, in some cases it was simultaneously detected in the pharyngeal and nasal exudates. The Enterobacteriaceae species, the group $\mathrm{C}$ streptococcus and the pneumococcus, were detected in sputum. Susceptibility tests were performed whenever the isolated strain was considered to be a factor in the infectious process, both for scientific purpose and the practical purpose of indicating therapeutic possibilities; ultimately, the clinician makes the choice of prescribing an antibiotic or not.

The research spanned from October 2011 through March 2012. The samples were collected with sterile swabs, in Amies medium tubes for preservation and transport [5]. The processing was conducted shortly after the sampling.

Besides the usually tested antibiotics, the research used several cephalosporins of all generations, as follows:

Cefuroxime axetil (CXA 30)

Third generation: Cefotaxime (CTX 30), Ceftazidime (CAZ 30), Cefpodoxime (CPD 30)

Association of a third generation cephalosporin with the beta-lactamase inhibitor clavulanic acid: Cefotaxime + clavulanic acid (CTC 40), Ceftazidime + clavulanic acid (CZC 40).

Fourth generation: Cefepime (FEP 30)

Table I - EUCAST breakpoint table

\begin{tabular}{|c|c|c|c|c|}
\hline \multirow[t]{2}{*}{ Cephalosporins } & \multirow{2}{*}{$\begin{array}{l}\text { Disk content } \\
(\mu \mathrm{g})\end{array}$} & \multicolumn{2}{|c|}{ Breakpoints $(\mathrm{mm})$} & \multirow[t]{2}{*}{ Observations } \\
\hline & & $\mathrm{S} \geq$ & $\mathrm{R}<$ & \\
\hline Cefuroxime & $30 \mu \mathrm{g}$ & 18 & 18 & \\
\hline Cefotaxime & $5 \mu \mathrm{g}$ & 20 & 16 & \\
\hline Ceftazidime & $10 \mu \mathrm{g}$ & 22 & 19 & \\
\hline Cefpodoxime & $10 \mu \mathrm{g}$ & 21 & 21 & \\
\hline Cefepime & $30 \mu \mathrm{g}$ & 24 & 21 & \\
\hline Cefepime with Clavulanic acid & $30 \mu \mathrm{g}$ & & & $\begin{array}{l}\text { The combination cefepime with clavulanic } \\
\text { acid lead to a growth inhibition zone } \\
\text { diameter of approx. } 5 \mathrm{~mm} \text {. }\end{array}$ \\
\hline Cefotaxime with Clavulanic acid & $40 \mu \mathrm{g}$ & & & Idem \\
\hline Ceftazidime with Clavulanic acid & $30 \mu \mathrm{g}$ & & & Idem \\
\hline
\end{tabular}


Association of a fourth generation of cephalosporins with clavulanic acid.

We used CLSI 2012 M100, S13 (M2-A11) standards, and EUCAST Breakpoint Table v. 2.0, valid from 2012-01-01 [6] (Table I).

According to 2012 Eucast standard, the susceptibility of Staphylococcus aureus to cephalosporins can be deduced from Cefoxitine sensitivity $(\mathrm{S} \geq 22, \mathrm{R}<22)$ and also Streptococcus B and $\mathrm{C}$ sensitivity to cephalosporins is inferred from benzyl-penicillin sensitivity. Within the present research we tested those species to cephalosporins.

\section{Results and Discussion}

The study is conducted on a total of 105 cases, 40 children and 65 adults.

The following bacterial species were isolated:

In children:

Staphylococcus aureus - 20 cases

Streptococcus pneumoniae - 7 cases

Streptococcus pyogenes ( $\beta$-hemolytic

Streptococcus group A) - 5 cases

Streptococcus $\beta$-hemolytic group C - 8 cases

In adults:

Staphylococcus aureus - 22 cases

Streptococcus pneumoniae - 5 cases

Klebsiella pneumoniae - 6 cases

Proteus - 4 cases

E.coli - 28 cases

Since the purpose of the study is to test the resistance to cephalosporins isolated strains, we presented overall results in antibiograms.

Staphylococcus aureus 42 strains

Streptococcus pneumonia 12 strains

Streptococcus $\beta$-hemolytic group C 8 strains

E.coli 28 strains

Klebsiella pneumonia 6 strains

Proteus 4 strains

We excluded $\beta$-hemolytic Streptococcus strains of group A, on which we did not perform an antibiogram. (Figure 1).
For bacterial strains isolated from patients with acute respiratory infections, we observed a significant resistance to Cefuroxime, a second generation cephalosporin in $47 \%$ of the cases, and between the third generation cephalosporins the resistance was highest in cefotaxime - ceftazidime $38 \%$ and $37 \%$ respectively.

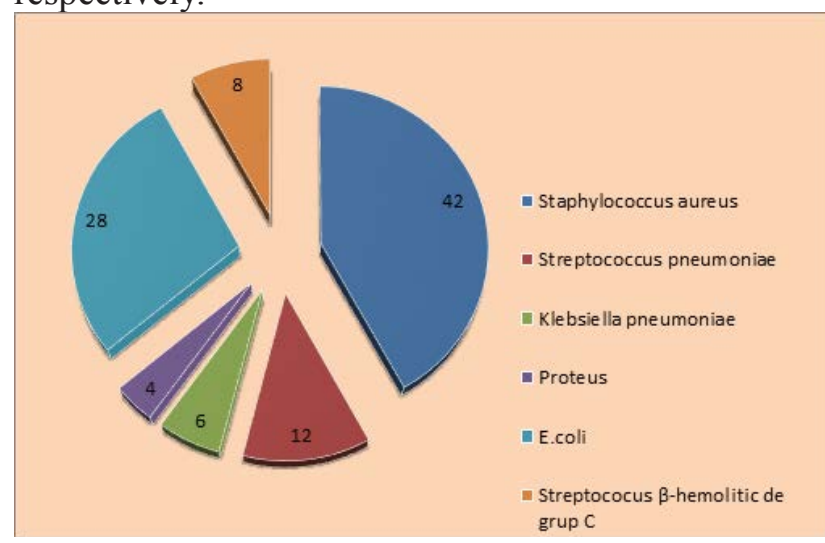

Figure 1 - Bacterial species isolated and tested to cephalosporins

Unlike the associations with beta-lactamase inhibitor, the resistance was much lower especially for ceftazidime + clavulanic acid association $(11 \%)$, compared to ceftazidime and also for the cefotaxime + clavulanic acid combination $(27 \%)$ compared to cefotaxime.

The lowest level of resistance to third generation cephalosporins has been tested against cefpodoxime $(10 \%)$. Cefepime, a fourth generation cephalosporin and clavulanic acid combination of cefepime was the most effective resistance to them being only $4 \%$ of the cases. (Figure 2).

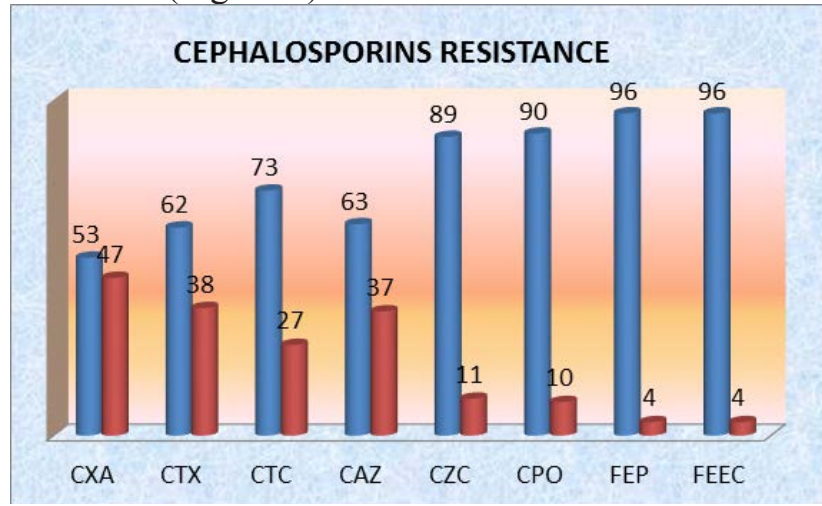

Figure 2 - Cephalosporin resistance in isolated strains (susceptible strains - blue, resistant strains - red) 
We observed that for Staphylococcus aureus, the number of the isolated strains, resistant to cefuroxime axetil was higher than those susceptible.

Although the first therapeutic indication for cefuroxime and cefuroxime axetil (oral dosage form) is an infection of the respiratory tract, such as acute and chronic bronchitis, bronchiectasis and infected bacterial pneumonia, it is necessary to select cases in which the infection is due to susceptible strains. (Figure 3)

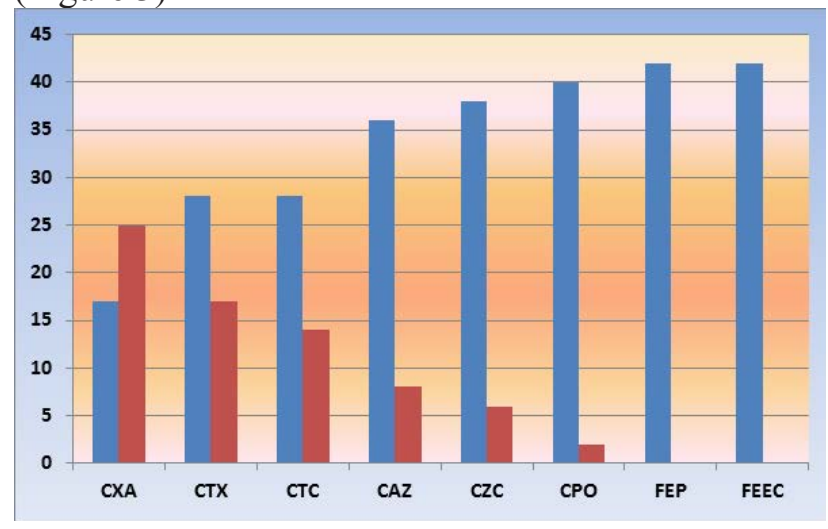

Figure 3 - Cephalosporin resistance in isolated strains (susceptible strains - blue, resistant strains - red)

There was also a significant number of strains resistant to cefotaxime, ceftazidime and cefotaxime + clavulanic acid. Resistance was low for cephoperasone, cefepime and cefepime + clavulanic acid. No strains were found resistant to the fourth generation cephalosporin (cefepime and cefepime + clavulanic acid combination).

Sensitivity to cephalosporins tested for Streptococcus pneumoniae was good because there were only 2 cases of resistance to cefuroxime.

In case of group $\mathrm{C}$ streptococci, we have obtained a number of strains resistant to cefuroxime, and a smaller number of cefotaxime and ceftazidime resistant cases. There was no resistance to clavulanic acid associations, which demonstrates resistance due to production of beta-lactamases. There was found a lower resistance to fourth generation cephalosporins.

E.coli presented the highest number of strains resistant to cefotaxime, ceftazidime and cefuroxime axetil. Compared to combinations with beta-lactamase inhibitor, we find a lower resistance, particularly in ceftazidime + clavulanic acid compared with ceftazidime. Resistance was also lower compared to cefpodoxime.

There were resistant E.coli strains at cefepime and cefepime + clavulanic acid.

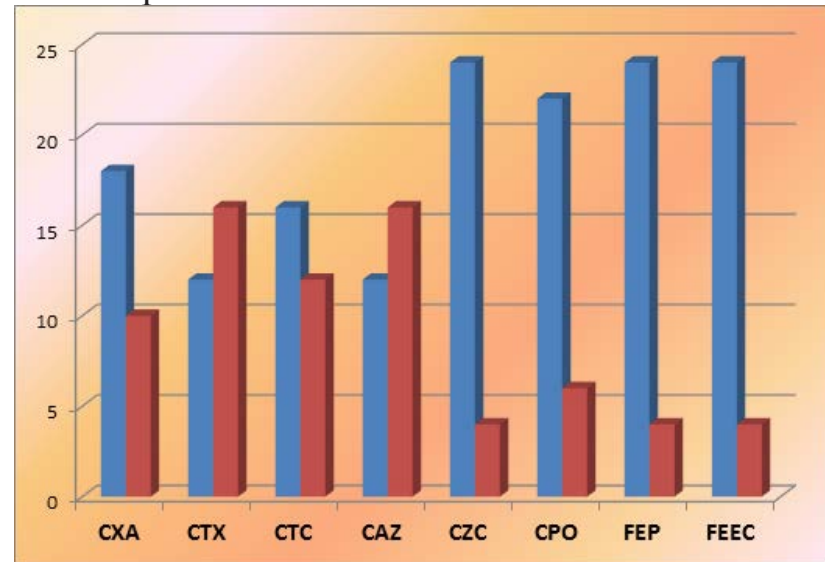

Figure 4 - Cephalosporin resistance in isolated strains (susceptible strains - blue, resistant strains - red)

Klebsiella presented strains resistant to cefuroxime, cefotaxime and cefpodoxime, and a small number of strains resistant to ceftazidime, as well as the ceftazidime - clavulanic acid and cefotaxime clavulanic acid combinations. The mechanism of the Gram-negative bacteria resistance to antibiotics mostly results from beta-lactamase and broad-specter beta-lactamase (ESBL) production, a fact sustained by the present results. No Klebsiella strains were found to be resistant to the fourth-generation cephalosporins tested.

The species pertaining to the Proteus genus were highly resistant to cefuroxim. No strain was resistant to the combinations between third-generation cephalosporins and clavulanic acid, nor to cefepim or the cefepim - clavulanic acid combination.

\section{Conclusions}

1. Resistance to cefuroxime, a secondgeneration cephalosporin, occurred for $47 \%$ of the cases. High frequency of resistance to cefuroxime was identified for Staphylococcus aureus, E.coli, K. 
pneumoniae and Proteus. Though the first therapeutic indication for cefuroxime and cefuroxime axetil (oral administration) consists in respiratory tract infections, the administered cephalosporins will reflect the antibiogram.

2. Of the bacterial species isolated from the acute respiratory tract infections, the highest resistance to third-generation cephalosporins is shown by E.coli and Klebsiella pneumoniae, both of which are resistant to cefotaxime and ceftazidime. The E.coli strains were more resistant in comparison with the results from previous researches, the latter having identified ceftazidime and cefotaxime resistance for $13 \%$ of the E.coli strains and $31 \%$ of the Klebsiella strains, while $25,8 \%$ presented ESBL-producing phenotype $[7,8,9]$.

3. Cefpodoxime can be considered a firstintention antibiotic in treating both upper and lower respiratory tract infections, as it produces the lowest level of resistance $-10 \%$ of the third-generation cephalosporins tested.

4. Cefepime is a fourth-generation cephalosporin which can be prescribed for severe respiratory tract infections, with strains resistant to other broad-specter antibiotics.

5. The resistance of the bacterial strains isolated within our research was very low at $4 \%$, and only occurred with E.coli. Gram positive cocci showed no resistance to cefepime.

6. The results confirm the low affinity for chromosomally encoded beta-lactamase, although there are certain resistant, broad-specter betalactamase producing strains (ESBL) that neutralize cefepime. The E.coli strains resistant to cefepime were also resistant to the cefepime - clavulanic acid combination.

\section{References}

1. Du B, I. (2003). Restriction of third-generation cephalosporin use decreases infection-related mortality. Crit Care Med. 31, 1088-1093
2. Hedrick, J.A. (2010). Community-acquired upper respiratory tract infections and the role of third-generation oral cephalosporins. Expert Rev Anti Infect Ther. 8(1), 15-21. DOI: 10.1586/ eri.09.115

3. Endimiani, A., Perez, F. \& Bonomo, R. (2008). Cefepime: reappraisal in an era of increasing antimicrobial resistance. Expert Rev Anti Infect Ther. 6, 805-824

4. Niculescu, C. \& Iordache, E. (2007). Recomandarea Academiei Americane de Pediatrie pentru prescrierea cefalosporinelor la pacienții alergici la penicilină. Craiova Medicală. 9(4)

5. Buiuc, D. \& Neguț, M. (1999). Tratat de Microbiologie Clinică. București: Ed. Medicală

6. CLSI 2012 M100, S13 ( M2-A11), EUCAST Breakpoint Table v. 2.0, valid from 2012-01-01

7. Chaudhary, M., Shrivastava, S.M., Varughese, L. \& Sehgal, R. (2008). Efficacy and safety evaluation of fixed dose combination of cefepime and amikacin in comparison with cefepime alone in treatment of nosocomial pneumonia patients. Curr Clin Pharmacol. 3, 118-122

8. Palladino, J.A., Sunderlin, J.L., Singer, M.E., Adelman, M.H. \& Schentag, J.J. (2008). Influence of extended-spectrum beta-lactams on gram-negative bacterial resistance. Am J Health Syst Pharm. 65, 1154-1159

9. Tumah, H.N. (2004). In vitro activity of cefepime and cefpirome compared to other thirdgeneration cephem antibiotics against gramnegative nosocomial pathogens. Pharmazie. 59, $854-858$ 